\title{
Timing behavior as a function of amount of reinforcement
}

\author{
Bernard Beer and Gerald Trumble \\ WALTER REED ARMY INSTITUTE OF RESEARCH AND UNIVERSITY OF MARYLAND
}

\begin{abstract}
Abstraet
One, two, or four food pellets were presented when a rat spaced its responses longer than $18 \mathrm{sec}$. The percentage of total responses which were reinforced was highest for the one-pellet condition and lowest for the four-pellet condition. Interresponse times were longest in the one-pellet condition.
\end{abstract}

\section{Problem}

Timing behavior has been studied in a free-operant situation by making the reinforcement contingent on the temporal spacing of responses (Wilson \& Keller, 1953). Typically, only responses occurring after a fixed time requirement are reinforced. This schedule of differential reinforcement of low response rates (DRL) characteristically generates an interresponse time (IRT) distribution in which the modal IRT's approximate the minimum time required for reinforcement.

In studies of deprivation and progressive satiation upon DRL behavior (Conrad et al., 1958), the major effects have occurred only with very shortdeprivations or at satiation. However, over the wide range of deprivation conditions little change in DRL performance was observed. Another study investigating the effects of liquid deprivation upon timing behavior (Mechner \& Guevrekian, 1962) used a slightly different procedure. In a two-lever situation rats were required to first press lever $A$, then after a minimum of 5 sec. to press lever B for a liquid reinforcement. The animals were tested over a water deprivation range of 4 to $56 \mathrm{hr}$. The results were similar to the study of Conrad et al., in that the deprivation conditions had little effect on the DRL behavior (A-B response distribution remained unchanged); however, increasing deprivation did shorten the pause between reinforcement and the next lever A response. Different results were obtained in an experiment using brain stimulation instead of water reinforcement (Kling \& Beer, 1962). "Efficiency" of DRL performance (total responses/total reinforcements) decreased linearly as the intensity of brain stimulation increased. This was the case only when all tested intensities were used in each daily session and changed frequently. However, when only one intensity was available during each experimental session little or no effect on DRL performance was observed. Since frequent reversals of deprivation conditions within each daily session is impractical the contrast between these states is minimal thereby lessening the effect of the motivational variables on DRL performance. The present study eliminates this difficulty by using size of food reinforcement rather than deprivation, thereby enabling the frequent contrast of this variable during each experimental session.

\section{Method}

The subjects were three naive male albino rats obtained from the Walter Reed Army Institute of Research rat colony. ${ }^{1}$ They weighed approximately 325 gm at the start of the study and were kept at $80 \%$ of their ad libitum weight. Water was continuously available in both the home cage and in the experimental chamber.

The testing chamber contained two plexiglas keys mounted on one wall, separated by a $3 / 4$ in thick and $11 / 2$ in long plexiglas barrier which prevented simultaneous manipulation of both keys. A light was mounted behind each key, a food magazine placed alongside one key, and a speaker housed in the opposite wall. Training and experimental conditions were programmed automatically by relay circuitry while responses on each key and response latencies between eath key press were recorded separately for each experimental condition. All behavior and stimulus conditions were continuously monitored by a cumulative and event recorder.

The subjects were initially trained to press Key B by reinforcing each response with a single $45 \mathrm{mgm}$ food pellet. They were then required to alternate responses, first key A then key B where each key alternation $(\mathrm{A}-\mathrm{B})$ produced a pellet. Stimulus lights behind each key were illuminated in turn designating the appropriate key. A time requirement was then introduced so that only those A-B responses longer than $18 \mathrm{sec}$. were reinforced and responses spaced less than $18 \mathrm{sec}$. reset the timing cycle which could only be restarted by another key A response. A-A or B-B responses had no effect. Three reinforcement sizes with accompanying auditory stimuli were then programmed. One food pellet was always available for correct $\mathrm{A}-\mathrm{B}$ responses during a $1 \mathrm{click} / \mathrm{sec}$. stimulus; two pellets during a five click/sec. presentation; and four pellets during a $10 \mathrm{click} / \mathrm{sec}$. trial. Each reinforcement size with its appropriate stimulus was available for $5 \mathrm{~min}$. after which the stimulus would change, thereby signaling the availability of another size. The sizes and their appropriate stimuli were each presented eight times in a random order throughout each daily session.

\section{Results and Diseussion}

"Efficiency" ratios (reinforced A-B responses/total A-B responses) were computed for each reinforcement size. These ratios clearly indicated a differential effect 


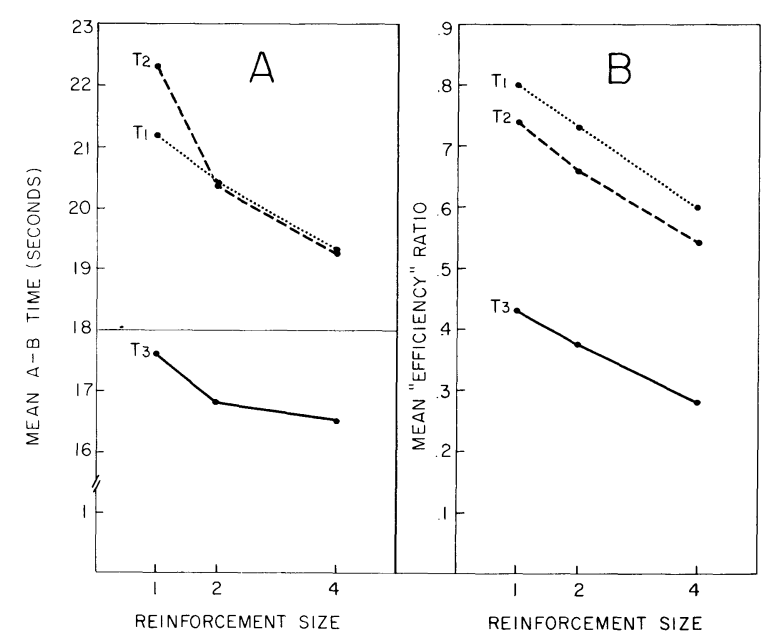

Fig. 1. Figure 1A shows the mean key A to key $B(A-B)$ response time for each animal as a function of the size of reinforcement. Figure 1B illustrates the mean "efficiency" ratio (reinforced responses/total A-B responses) for each reinforcement size.

of reinforcement size on timing behavior (Fig. 1B). For all animals there existed a negative linear relationship between size of reinforcement and "efficiency" ratio. The "efficiency" ratio for the four-pellet condition consistently remained lower and never equaled or exceeded the ratio for the one-pellet condition. A correlate of these results can be found in the analysis of the mean A-B time. Figure 1A shows the effect of food size on the delay of responding. Responses during the one-pellet condition are delayed the longest, while the shortest response delays are manifested during the four-pellet size. The line drawn at $18 \mathrm{sec}$. indicates the interval above which reinforcements may be obtained. Individual records of interresponse time (IRT) distributions duplicate these results. They consistently showed the modal IRT peaking later in the smallest reinforcement size and earlier during the largest size. Measures of response variability indicated the least variability during the four-pellet and the most during the onepellet condition (one-pellet, Sigma $=.98$; two-pellet, Sigma $=.69$; four-pellet, Sigma $=.54$ ). It is clear from these data that DRL behavior is sensitive to changes in the amount of reinforcement. Continual and repeated exposure to the motivational variables might be the explanation of the present study's contrasting results with the deprivation experiments. The amount of reinforcement rather than deprivation level and the contrast of motivational conditions within a session are the major procedural differences between the studies. Additional support for the contrast variable comes from the Kling and Beer experiment where similar results to the present study were seen only when the motivational variable was manipulated often in a single session. Their data along with the present study suggest that if one were able to frequently reverse the level of deprivation within a session similar changes in DRL behavior would ensue.

\section{Relerences}

CONRAD, D. G., SIDMAN, M., \& HERRNSTEIN, R. J. The effects of deprivation upon temporally spaced responding. J. exp. Anal. Behav., 1958, 1, 59-65.

KLING, J. W., \& BEER, B. Timing behavior as a function of current and locus of intracranial stimulation. Eastern Psychological Association meetings, 1962.

MECHNER, F., \& GUEVREKIAN, L. Effects of deprivation upon counting and timing in rats. J. exp. Anal. Behav., 1962, 4, 463-467.

WILSON, M. P., \& KELLER, F. S. On the selective reinforcement of spaced responses. J. comp. physiol. Psychol., 1953, 46, 190-193. Note

1. The principles of laboratory animal care as promulgated by the National Society for Medical Research were observed. 